\title{
An Existence Theorem in the Calculus of Variations Based on Sobolev's Imbedding Theorems
}

\author{
E. H. RoTHE \\ Communicated by L. CESARI
}

\section{Introduction}

This paper deals with the minimum problem concerning an integral of the form

$$
I(x)=\int_{\Omega} f\left(t, x, x^{(1)}, \ldots, x^{(m)}, \ldots, x^{(l)}\right) d t
$$

where $l$ is an arbitrary positive integer, where $x^{(m)}$ denotes the vector $x^{(m)}=$ $\left(x_{1}^{(m)}, \ldots, x_{\sigma_{m}}^{(m)}\right)$ of the derivatives of $x$ of order $m$ taken in an arbitrary but fixed order, and where $\Omega$ is a bounded open domain in the $n$-dimensional Euclidean space $E^{n}$ of points $t=\left(t_{1}, \ldots, t_{n}\right)$.

In certain basic aspects (e.g. in the use of convexity considerations and of the reflexitivity of the Sobolev spaces) the method of the present paper is the same as the one used in a recent paper by F. E. BROWDER [3] ${ }^{1}$, while in other aspects the treatment is different. This will be clear from the following outline of the existence proof given in the present paper:

The Sobolev space $W_{p}^{l}=W_{p}^{l}(\Omega)$ is a reflexive Banach space. Therefore, the closed ball $B_{R} \subset W_{p}^{l}$ with radius $R$ and center 0 is weakly compact, i.e. compact in the relative topology of $B_{R}$ induced by the weak topology of $W_{p}^{l}$. Consequently for the proof of the existence of an $x_{0} \in B_{R}$ minimizing $I(x)$ in $B_{R}$ it will be sufficient to show that $I(x)$ is weakly lower semi-continuous (see [10]). To do this, we use the notation

$$
\begin{gathered}
f(x ; y)=f\left(t, x^{(1)}, \ldots, x^{(l-1)}, y^{(l)}\right), \\
I(x ; y)=\int_{\Omega} f(t, x(t) ; y(t)) d t
\end{gathered}
$$

such that

$$
I(x)=I(x ; x) .
$$

1 Numbers in brackets refer to the bibliography at the end of the paper. 
Under assumptions to be specified later the following statements $\alpha$ ) and $\beta$ ) will be proved:

$\alpha$ ) for fixed $x_{0} \in B_{R}, I_{0}(y)=I\left(x_{0} ; y\right)$ is weakly lower semi-continuous in $y$,

$\beta$ ) for fixed $y \in B_{R}, I(x ; y)$ is weakly continuous in $x$.

As to the proof of $\alpha$ ) a nearly obvious argument (given in detail in [11; theorem 4.1]) shows that it will be sufficient to prove: to each, $y_{0}$ in $B_{R}$ there exists a bounded linear functional $l_{0}=l_{0}(y)$ on $W_{p}^{l}$ such that

$$
I_{0}(y)-I_{0}\left(y_{0}\right) \geqq l_{0}\left(y-y_{0}\right), \quad y, y_{0} \in B_{R} .
$$

The main assumption for the proof of (1.5) will be a convexity assumption on $f$ with respect to the highest derivatives which implies a corresponding convexity of $I(\mathrm{x} ; y)$ with respect to $y^{2}$. A linear functional $l_{0}$ satisfying $(1.5)$ is then obtained as follows: if (for $x$ fixed) $\Pi$ denotes the product space of $W_{p}^{l}$ with the real line consisting of couples $u=(y, r)$, then the set $\Pi_{1} \subset \Pi$ above the graph $r=I_{0}(y)$, i.e. the set of points $(y, r)$ with $r \geqq I_{0}(y)$ is convex and has therefore under rather general conditions a closed supporting hyperplane at the point $u_{0}=\left(y_{0}, I_{0}\left(y_{0}\right)\right)$ given by an equation of the form

$$
h_{0}(u)=h_{0}\left(u_{0}\right)
$$

where $h_{0}$ is a continuous linear functional on $\Pi$. It is then proved that the restriction of $h_{0}$ to $W_{p}^{l}$ multiplied by a proper constant is an $l_{0}$ of the desired properties (section 3).

The proof of the statement $\beta$ ) above is given in section 4 . It is based on the extended form (4.2) of the Friedrichs-Sobolev inequality (4.1). The latter is proved in Appendix A by applying SoBolev's imbedding theorems. The existence of a minimizing $x_{0} \in B_{R}$ then follows easily by combining the results of sections 3 and 4 (section 5 ).

The inequality (4.1) is a generalization of the Friedrichs inequality given for the case $l=1$ in an inner product space [4; p.489]. (See also [7; section 5] for a more general case.) From the point of view taken in the present paper an essential feature of FRIEDRICH's inequality and its generalization (4.2) is that they immediately imply the weak continuity of the $L_{p}$ norm of the derivatives of order $<l$ of elements $x \in B_{R}$. (See Remark 1 at the end of section 4.)

The compactness theorem known as Rellich's lemma (Rellichscher Auswahlsatz) $[4 ;$ p.489] is a direct consequence of FRIEDRICH's original inequality. Correspondingly it is shown in Appendix $B$ that inequality (4.2) implies immediately a compactness theorem which may be considered as a generalization of RELLICH's lemma. This theorem is not new. It is a special case of KondRaSEv's theorem on the complete continuity of the SoBoLEV imbedding operator [13; chapter I, $\S 11]$.

${ }^{2}$ Instead of a convexity assumption, a positivity assumption on the second differential (implying convexity) was used in [11]. The author is indebted to G. MINTY for pointing out in conversations that convexity would be sufficient. Moreover in that paper in which the case $l=1$ was treated the assumption just mentioned was made not only with respect to the first derivatives of $x$ but also with respect to $x$ itself. This resulted in a theorem of unnecessarily narrow scope as was pointed by L.M. Graves [Math. Reviews 15, 39 (1954)]. 


\section{Preliminaries}

$\Omega$ will always be a bounded open domain in $E^{n}$. Such an $\Omega$ is called a Sobolev domain if the Sobolev imbedding theorems $[13$; pp. 56, 57] hold. Sufficient conditions for this to be the case may be found in $[13 ; \mathrm{p} .66]$ or [12; chapter IV].

By "derivative" of a function $x=x(t)$ defined in $\Omega$ we will always mean generalized derivative as defined in $[13 ; \mathrm{p} .33]$. The $L_{p}$ spaces and the norm $\|x\|_{p}$ of an $x \in L_{p}$ are defined as usual. We always assume $p>1$.

For any positive integer $l$ the Banach space $W_{p}^{l}=W_{p}^{l}(\Omega)$ is then defined as the space of all $x \in L_{p}(\Omega)$ which have derivatives in $L_{p}(\Omega)$ of order up to and including $l$, while the norm of $x$ is defined by

$$
\|x\|_{W_{p}^{l}}=\left(\|x\|_{p}^{p}+\|x\|_{i, p}^{p}\right)^{1 / p} .
$$

Here $\|x\|_{l, p}$ is defined as follows: let $\|x\|_{E}$ denote the Euclidean norm of the vector $x^{(l)}$ defined in the first paragraph of the introduction; then

$$
\|x\|_{l, p}=\left(\int_{\Omega}\left\|x^{(l)}\right\|_{E}^{p} d t\right)^{1 / p} .
$$

Theorem 2.1. The Banach space $W_{p}^{l}$ is reflexive.

Proof. If the norm $\|x\|_{l, p}$ were re-defined by replacing in its definition (2.2) the Euclidean norm $\|x\|_{E}$ by the $p$ norm, i.e. by

$$
\|x\|=\left[\sum_{i=1}^{l_{\sigma}}\left(x_{i}^{(l)}\right)^{p}\right]^{1 / p},
$$

then the proof would be the same as the one given for theorem 2.1 in [11]. (Cf. also [2; p.863] and [3] where this norm is used.) However a perusal of that proof makes clear how it should be modified to meet our case by using the following:

Lemma 2.1. For any positive s let $\|y\|_{E_{s}}$ be the Euclidean norm of $y=\left(y_{1}, \ldots, y_{s}\right)$. Then the space of all vector functions $y(t)=\left(y,(t), \ldots, y_{s}(t)\right)$ for which $\|y(t)\|_{E_{s}} \in L_{p}$ with norm

is uniformly convex.

$$
\|y\|=\left\{\int_{\Omega}\|y(t)\|_{E_{s}}^{p} d t\right\}^{1 / p}
$$

Proof. Since $L_{p}$ is uniformly convex, it is easily seen that the proof given by M.M.DAY for his theorem 3 in [5] is valid for the present case. (Cf. also the concluding remarks in [5].)

We now specify the assumptions on the integrand $f$ of the integral (1.1). $t$ and $\sigma_{m}(m=0,1, \ldots, l)$ are defined as in the introduction, and $p^{(m)}$ is a vector with components $p_{1}^{(m)}, \ldots, p_{\sigma_{m}}^{m}$. Then the function $f\left(t, p^{(0)}, \ldots, p^{(l)}\right)$ is supposed to satisfy the following four conditions:

A) $f$ satisfies a Hoelder (or Lipschitz) condition with respect to the variables $p^{(0)}, p^{(i)}, \ldots, p^{(l-1)}$.

B) $f$ is convex with respect $p^{(l)}$.

C) For any couple $x, y$ of elements of $B_{2 R}, f(x ; y)$ (see (1.2)) exists and is integrable over $\Omega$.

Arch. Rational Mech. Anal., Vol. 21 
D) To every couple $x_{0}, y_{0}$ in $B_{R}$ there exists a neighborhood $U\left(y_{0}\right)$ of $y_{0}$ such that $I\left(x_{0} ; y\right)$ is bounded from above for $y \in U\left(y_{0}\right)$.

Remark. D) is satisfied if to every couple $x_{0}, y_{0}$ of elements of $B_{R}$ there exists a constant $C=C\left(x_{0}, y_{0}\right)$ and a neighborhood $U\left(y_{0}\right)$ of $y_{0}$ such that for each $t \in \Omega$

$$
f\left(x_{0}, y\right)<C\left\|y^{(l)}\right\|_{E}^{p} \text { for } y \in U\left(y_{0}\right) .
$$

\section{Proof of the lower semicontinuity of $I_{0}(y)=I\left(x_{0} ; y\right)$}

As already pointed out it is sufficient to prove that (1.5) holds for some bounded linear functional $l_{0}$. To prove the existence of such a functional we note first that the convexity assumption $\mathrm{B}$ ) on $f$ implies immediately the convexity of $I_{0}(y)$, and that assumption D) implies that $I_{0}(y)$ is locally bounded from above. It now follows from a well known theorem [1; Chapter II, $\S 5$, proposition 2] that $I_{0}(y)$ is continuous in $B_{R}$. From this it is easily seen that the set $\Pi_{1} \subset \Pi$ defined in the introduction contains interior points (namely all points $\left(y_{0}, r_{0}\right)$ with $r_{0}>I_{0}\left(y_{0}\right)$; see also [9]), and that the graph of $I_{0}$ belongs to the boundary of $\Pi_{1}$.

From these properties we conclude by a well known theorem [I; chapter II, $\S 3$, proposition 3] that every point $u_{0}=\left(y_{0}, I_{0}\left(y_{0}\right)\right)$ of the graph of $I_{0}$ admits a closed supporting hyperplane $H_{0}$. Therefore there exists a continuous linear functional $h_{0}=h_{0}(u)$ on $\Pi$ such that

$$
h_{0}(u)=h_{0}\left(u_{0}\right)
$$

is an equation for $H_{0}$ while

$$
h_{0}(u) \geqq h_{0}\left(u_{0}\right) \text { for } u \in \Pi_{1} .
$$

Now let $e_{0}$ be an arbitrary element of $\Pi$ which is not an element of $W_{p}^{l}$. Then every $u \in \Pi$ has the unique representation

$$
u=r e_{0}+y, \quad r \text { real, } y \in W_{p}^{l} .
$$

Thus

$$
h_{0}(u)=r h_{0}\left(e_{0}\right)+h_{0}(y) .
$$

Since the points of the graph of $I_{0}$ are characterized by $r=I_{0}(y)$, we see from (3.4) that

$$
h_{0}(u)=I_{0}(y) h_{0}\left(e_{0}\right)+h_{0}(y)
$$

for $u$ on the graph. But since the graph belongs to $\Pi_{1}$ we see from (3.2) that

or

$$
I_{0}(y) h_{0}\left(e_{0}\right)+h_{0}(y) \geqq I_{0}\left(y_{0}\right) h\left(e_{0}\right)+h\left(y_{0}\right)
$$

$$
\left(I_{0}(y)-I_{0}\left(y_{0}\right)\right) h_{0}\left(e_{0}\right) \geqq-h_{0}\left(y-y_{0}\right) .
$$

If now

$$
h_{0}\left(e_{0}\right) \neq 0,
$$

then (3.5) implies (1.5) if we take for $l_{0}$ the restriction of the linear functional $-h_{0} / h_{0}\left(e_{0}\right)$ to $W_{p}^{l}$. 
Suppose now $h_{0}\left(e_{0}\right)=0$. Then $e_{0}$ is an element of the hyperspace $H$ given by $h_{0}(u)=0$. Since $e_{0}$ by definition $\notin W_{p}^{l}$, the two hyperspaces $H$ and $W_{p}^{l}$ of $\Pi$ are not identical, and $W_{p}^{l}$ contains an element $u_{1}$ which is not in $H$, i.e. for which $h_{0}\left(u_{1}\right) \neq 0$. We now set $e_{1}=e_{0}+u_{1}$. Then $h_{0}\left(e_{1}\right)=h_{0}\left(e_{0}\right)+h_{0}\left(u_{1}\right)=h_{0}\left(u_{1}\right) \neq 0$, i.e. (3.6) is satisfied with $e_{0}$ replaced by $e_{1}$. On the other hand, $e_{1} \notin W_{p}^{l}$ since otherwise $e_{0}=e_{1}-u_{1}$ would be in $W_{p}^{l}$.

\section{The weak continuity of $I(x ; y)$ with respect to $x$}

The goal of this section is the proof of

Theorem 4.1. For fixed $y_{0} \in B_{R}, I\left(x ; y_{0}\right)$ is weakly continuous in $x$ in the weak topology of $B_{R}$. The continuity is uniform as $y_{0}$ varies over $B_{R}$.

The proof will be based on

Theorem 4.2. Let $\Omega$ be a Sobolev domain. Then there exists a positive constant $M=M(\Omega)$ with the following property: if for any positive $\delta, I_{\delta} \subset \Omega$ denotes a cube of sidelength $\delta$, and if for any positive integer $N, \Omega_{N}=\Omega_{N}(\delta)$ denotes the union of $N$ such cubes $I_{\delta}^{\prime}, \ldots, I_{\delta}^{N}$ with disjoint interiors, then for $m=0,1, \ldots, l-1$ there exist for $\delta<1$ an $\Omega_{N}$ and bounded linear functionals $\lambda_{\beta_{1} \ldots \beta_{n}}^{j}$ on $W_{p}^{l}(j=1, \ldots, N)$, $\sum_{i} \beta_{i}=k$ where $k$ varies from 0 to $l-m-1$ such that the following FriedrichsSobolev inequality holds:

$$
\begin{aligned}
& \int_{\Omega_{N}}\left|\frac{\partial^{m} x}{\partial t_{1}^{m_{1}} \ldots \partial t_{n}^{m_{n}}}\right|^{p} d t \\
& \leqq M^{p} \delta^{(l-m) p} \int_{\Omega_{N}}\left[\sum_{\Sigma \beta_{i}=l-m}\left(\frac{\partial^{l} x}{\partial t_{1}^{m_{1}+\beta_{1}} \ldots \partial t_{n}^{m_{n}+\beta_{n}}}\right)^{2}\right]^{p / 2} d t+ \\
&+\delta^{-m p} \sum_{j=1}^{N} \sum_{k=0}^{l-m-1}\left[\sum_{\Sigma}\left(\lambda_{\beta_{l}=k}^{j} \ldots \beta_{n}(x)\right)^{2}\right]^{p / 2} \quad\left(\sum m_{i}=m\right) .
\end{aligned}
$$

The proof will be given in Appendix A.

Our next goal is the proof of

Theorem 4.3. Let $\eta$ be a given positive number. Then (with the notations used in theorem 4.2) there exist a positive $\delta<1$ (which may be chosen arbitrarily small) and bounded linear functionals $\lambda_{\beta_{1} \ldots \beta_{n}}^{j}$ on $W_{p}^{l}$ such that for $x \in B_{2 R} \subset W_{p}^{l}$

$$
\begin{aligned}
\int_{\Omega}\left|\frac{\partial^{m} x}{\partial t_{1}^{m_{1}} \ldots \partial t_{n}^{m_{n}}}\right|^{p} d t & \\
\leqq & M^{p} \delta^{(l-m) p} \int_{\Omega}\left[\sum_{\Sigma \beta_{i}=l-m}\left(\frac{\hat{o}^{l} x}{\partial t_{1}^{m_{1}+\beta_{1}} \ldots \partial t_{n}^{m_{n}+\beta_{n}}}\right)^{2}\right]^{p / 2} d t+ \\
& +\delta^{-m p} \sum_{j=1}^{N} \sum_{k=0}^{m-l-1}\left[\sum_{\sum \beta_{i}=k}\left(\lambda_{\beta_{1} \ldots \beta_{n}}^{j}(x)\right)^{2}\right]^{p / 2}+\eta
\end{aligned}
$$


Moreover, the integral at the right member of this inequality may be replaced by $(2 R)^{p}$.

The proof is based on the next three lemmas.

Lemma 4.1. There exists $a q>p$ and a constant $C=C(q)$ such that for each $x \in W_{p}^{l}$

$$
\left\|x_{v}^{(m)}\right\|_{q} \leqq C\|x\|_{W_{p}}
$$

for $m=0,1, \ldots, l-1$ and $v=1, \ldots, \sigma_{m}$. (For the notation see the first paragraph of the introduction.)

Proof. Suppose first that $m \geqq l-n / p$. Then according to a result by SoBolev [13, Theorem, p.69], (4.3) is true for every positive $q<n p /(n-(l-m) p)$. Since the latter number is greater than $p$, the lemma is proved under the assumption made.

If however $m<l-n / p$, then by the same theorem $x_{v}^{(m)}$ is continuous, and moreover $\left|x_{v}^{(m)}\right| \leqq$ const $\|x\|_{W_{p}^{l}}$. This inequality obviously implies (4.3) for every positive $q$ since the measure of $\Omega$ is finite.

Lemma 4.2. Let $0<p<q$. Let $\propto$ be a positive number, and $\mathfrak{F}=\{z\}$ a family of elements of $L_{q}(\Omega)$ for which

$$
\int_{\Omega}|z|^{q} d t<\alpha
$$

then there corresponds to each positive $\eta$ a $\zeta$ such that

$$
\int_{\mathbb{E}}|z|^{p} d t<\eta, \quad z \in \mathfrak{F}
$$

if, with $\mu(E)$ denoting the measure of the set $E$,

$$
\mu(E)<\zeta, \quad E \in \Omega .
$$

Proof. Setting $z_{1}=x^{p}$, one sees that it is sufficient to consider the case $p=1$, and a proof in this case is given in [8; p.163].

Lemma 4.3. With the notation of theorem 4.2 there corresponds to each positive $\eta$ an $\Omega_{N}=\Omega_{N}(\delta)$ (with arbitrarily small $\delta$ ) such that for each $x \in B_{R} \subset W_{p}^{l}$

$$
\int_{\Omega-\Omega_{N}}\left|x_{v}^{(m)}\right|^{p} d t<\eta \quad(m=0,1, \ldots, l-1) .
$$

Proof. We first choose a $q$ according to lemma 4.1. We then see from (4.3) that (4.4) is satisfied with $\mathfrak{F}=\left\{x_{v}^{(m)} \mid x \in B_{R}\right\}$ and with $\alpha=C^{q} R^{q}$. On the other hand since $\Omega$ is a bounded open set, there exists an $\Omega_{N}(\delta)$ with arbitrarily small $\delta$ such that (4.6) is true with $E=\Omega-\Omega_{N}$. Thus our assertion follows from lemma 4.2.

Proof of Theorem 4.3. (4.2) is now an immediate consequence of theorem 4.1 and lemma 4.3. The additional assertion of our theorem follows from the fact that integral at the right member of (4.2) is not greater than $\|x\|_{l, p}^{p} \leqq\|x\|_{w_{p}^{l}}$ as is seen from definitions (2.1) and (2.2). 
We are now in a position to prove theorem 4.1. In obvious notation we have for $x, a, y_{0} \in B_{R}$

$$
\begin{aligned}
& I\left(x ; y_{0}\right)-I\left(a ; y_{0}\right) \\
& =\int_{\Omega} \sum_{m=0}^{l-1}\left[f\left(t, a^{0}, a^{1}, \ldots, a^{(m-1)}, x^{(m)}, x^{(m+1)}, \ldots, x^{(l-1)}, y_{0}^{l}\right)-\right. \\
& \\
& \left.\quad-f\left(t, a^{0}, a^{1}, \ldots, a^{(m-1)}, a^{(m)}, x^{(m+1)}, \ldots, x^{(l-1)}, y_{0}^{l}\right)\right] d t
\end{aligned}
$$

From the definition of the vector $x^{(m)}$ (see the first paragraph of the introduction) we see that the bracket in (4.8) can be written as a sum of differences in each of which only one component $a_{v}^{(m)}$ changes to $x_{v}^{(m)}$. From assumption A) we conclude therefore that the right member of (4.8) is majorized by a finite number, say $\sigma$, terms of the form

$$
L \int_{\Omega}\left|\frac{\partial^{m}(x-a)}{\partial t_{1}^{m_{1}} \ldots \partial t_{n}^{m_{n}}}\right|^{\alpha} d t, \quad \sum m_{i}=m
$$

where $L$ and $\alpha$ are positive constants, the latter $\leqq 1$. Since $p>1$, it is easily seen from HOELDER's inequality that the expression (4.9) is not greater than

$$
\left\{L_{1} \int_{\Omega}\left|\frac{\partial^{m}(x-a)}{\partial t_{1}^{m_{1}} \ldots \partial t_{n}^{m_{n}}}\right|^{p} d t\right\}^{\alpha / p}, \quad L_{1}=L^{p / \alpha}(\mu(\Omega))^{(p-\alpha) / \alpha}
$$

where $\mu(\Omega)$ denotes the measure of $\Omega$.

Now $x$ and $a$ are elements of $B_{R}$. Therefore $x-a \in B_{2}$ and we may apply theorem 4.3 to the integral in (4.10) with $x$ replaced by $x-a$. We see from (4.8) and (4.10) that with $\sigma_{1}=\sigma^{p / \alpha}$

$$
\begin{aligned}
\left|I\left(x ; y_{0}\right)-I\left(a ; y_{0}\right)\right|^{p / \alpha} \leqq & \sigma_{1} L_{1} \eta+\sigma_{1} L_{1}\left(M \delta^{l-m} 2 R\right)^{p}+ \\
& +\sigma_{1} L_{1} \delta^{-m p} \sum_{j=1}^{N} \sum_{k=0}^{l-m-1}\left[\sum_{\Sigma \beta_{i}=k}\left(\lambda_{\beta_{1} \ldots \beta_{n}}^{j}(x-a)\right)^{2}\right]^{p / 2} .
\end{aligned}
$$

Now if $\varepsilon$ is a given positive number, we first choose $\eta$ such that the first term of the right member of (4.11) is less than $\varepsilon / 3$, then $\delta$ such that the second term is also majorized by $\varepsilon / 3$. After $\eta$ and $\delta$ are fixed, the third term of the right member of (4.11) is a finite sum of powers of bounded linear functionals. It is therefore clear that there exists a weak neighborhood $U(a)$ such that this term is also not greater then $\varepsilon / 3$ for $x \in U(a)$. This obviously proves theorem 4.1.

Remark 1. The argument at the end of the proof just given shows also that for $x \in B_{R}$ and for $m=0,1, \ldots, l-1$ the $L_{p}$ norm of any derivative $x_{v}^{(m)}$ of $x$ is continuous in the weak topology of $B_{R}$. Indeed, the absolute value of $\left\|x_{v}^{(m)}\right\|_{p}-$ $\left\|a_{v}^{m}\right\|_{p}$ is not greater than $\left\|(x-a)_{v}^{m}\right\|_{p}$, and $\left\|(x-a)_{v}^{(m)}\right\|^{p}$ is the integral in (4.10), and therefore by theorem 4.3 is majorized by the right member of (4.11) with $\sigma_{1}$ and $L_{1}$ replaced by 1 .

Remark 2. For $x$ in $B_{R}$ the norm $\|x\|_{l, p}$ (defined by (2.2)) is lower semicontinuous in the weak topology of $B_{R}$. Indeed, since a Banach space norm $\|x\|_{w_{p}^{1}}$ has this property [10; lemma 2.4], and on account of (2.1), our assertion follows from remark 1 (with $m=0$ ). 


\section{Proof of the existence of an $x_{0} \in B_{R}$ minimizing the integral (1.1)}

As pointed out in the introduction, it is sufficient to prove the weak lower semicontinuity of $I(x)$. Let $\varepsilon$ be a given positive number. For $x$, $a$ in $B_{R}$ we see from (1.4) that

$$
I(x)-I(a) \geqq I(a ; x)-I(a ; a)-|I(x ; x)-I(a ; x)| .
$$

Now since $I(a ; x)$ is weakly lower semicontinuous in $x$ (section 3 ), there exists a weak neighborhood $V(a)$ such that the first difference of the right member of (5.1) is not smaller than $-\varepsilon$ for $x \in V(a)$, and by theorem 4.1 there exists a weak neighborhood $U(a)$ such that $|I(x ; x)-I(a ; x)|<\varepsilon$ for $x \in U(a)$. Thus we see from (5.1) that $I(x)-I(a) \geqq-2 \varepsilon$ for $x \in V(a) \cap U(a)$.

\section{Appendix A}

Proof of theorem 4.2. We first consider the cube $I=I_{1} \subset E^{n}$ of side length 1 with the origin as center and with sides parallel to the coordinate axes. The norm $\|x\|_{W_{p}^{\prime}(I)}$ of $x \in W_{p}^{l}(I)$ is then defined in section 2 with $\Omega$ replaced by $I$. With SOBOLEV we introduce a new norm $\|x\|_{W_{p}^{2}}^{0}$ in the following way: Let $S^{l}$ be the vector space of all polynomials

$$
P=\sum_{k=0}^{l-1} \sum_{\sum \alpha_{i}=k} a_{\alpha_{1}} \ldots \alpha_{n} t_{1}^{\alpha_{1}} \ldots t_{n}^{\alpha_{n}}
$$

of degree less than $l$ in the variables $t_{1}, \ldots, t_{n} . S^{l}$ may be considered a finite dimensional subspace of the linear space underlying $W_{p}^{l}(I)$. Therefore there exists a "supplementary" space $L_{p}^{l}$ such that every $x \in W_{p}^{l}(I)$ has the unique representation

$$
x=P+x_{1}, \quad P \in S^{l}, \quad x_{1} \in L_{p}^{l} .
$$

Then with the norm \|\|$_{l, p}$ defined by (2.2) and with $\|P\|_{S^{i}}$ given by

$$
\|P\|_{S^{I}}=\left\{\sum_{k=0}^{l-1}\left[\sum_{\Sigma \alpha_{i}=k}\left(a_{\alpha_{1}} \ldots \alpha_{n}\right)^{2}\right]^{p / 2}\right\}^{1 / p}
$$

the new norm is defined by

$$
\|x\|_{W_{p}^{l}}^{0}=\left\{\|P\|_{S^{i}}^{p}\left\|+x_{1}\right\|_{l, p}^{p}\right\}^{1 / p}
$$

which may also be written as

$$
\|x\|_{W_{p}^{l}}^{0}=\left\{\|P\|_{S^{1}}^{p}+\|x\|_{i, p}^{p}\right\}^{1 / p}
$$

since by (A.2) the $l^{\text {th }}$ derivatives of $x$ agree with those of $x_{1}$, and therefore $\|x\|_{l, p}=$ $\left\|x_{1}\right\|_{l, p}$.

Now $I$ is clearly a Sobolev domain. Therefore the following result of SOBOLEV is valid $[13 ;$ p. 72$]$ :

Theorem A.1. The norms $\|x\|_{W_{p}^{l}(I)}$ and $\|x\|_{W_{p}^{l}(I)}^{0}$ are equivalent, i.e. there exist two positive constants $m$ and $M$ (not depending on $x$ ) such that

$$
m\|x\|_{W_{p}^{i}(I)}^{0} \leqq\|x\|_{W_{p}^{l}(I)} \leqq M\|x\|_{W_{p}^{l}(I)}^{0} .
$$


Since $\|x\|_{p} \leqq\|x\|_{W_{p}^{l}(I)}(\operatorname{see}(2.1))$, we conclude from (A.6) that $\|x\|_{p} \leqq M\|x\|_{W_{p}^{l}(I)}^{0}$, or written out

$$
\begin{aligned}
\int_{I}\|x\|^{p} d t \leqq & M^{p}\left\{\int_{I}\left[\sum_{\Sigma \alpha_{i}=l}\left(\frac{\partial^{l} x}{\partial t_{1}^{\alpha_{1}} \ldots \partial t_{n}^{\alpha_{n}}}\right)^{2}\right]^{p / 2} d t+\right. \\
& \left.+\sum_{k=0}^{l-1}\left[\sum_{\sum \alpha_{i}=k}\left(a_{\alpha_{1} \ldots \alpha_{n}}(x)\right)^{2}\right]^{p / 2}\right\}, \quad x \in W_{p}^{l}(I) .
\end{aligned}
$$

Here we consider the $a_{a_{1} \ldots a_{n}}$ as bounded linear functionals on $W_{p}^{l}(I)$; this is possible since by (A.1) these coefficients are certainly bounded linear functionals on the finite dimensional space $S^{l}$ which by the Hahn-Banach theorem may be extended to $W_{p}^{l}(I)$.

We now want to extend (A.7) to the derivatives $x_{v}^{(m)}$ of $x(m=0,1, \ldots, l-1$; $\left.v=1, \ldots, \sigma_{m}\right)$. Since the $(l-m)^{\text {th }}$ derivatives of $x_{v}^{(m)}$ are $l^{\text {th }}$ derivatives of $x$ we see that $x_{v}^{(m)} \in W_{p}^{l-m}(I)$. Therefore (A.7) remains valid if we replace $x$ by $x_{v}^{(m)}$ and $l$ by $l-m$, and the $a_{\alpha_{1} \ldots \alpha_{n}}$ by appropriate linear functionals on $W_{p}^{l-m}(I)$. Thus for

we obtain

$$
x_{v}^{(m)}=\frac{\partial^{m} x}{\partial t_{1}^{m_{1}} \ldots \partial t_{n}^{m_{n}}}, \quad \sum_{\rho=1}^{n} m_{\rho}=m
$$

$$
\begin{aligned}
\int_{I}\left|\frac{\partial^{m}}{\partial t_{1}^{m_{1}} \ldots \partial t_{n}^{m_{n}}}\right|^{p} d t & \leqq M^{p} \int_{I}\left[\sum_{\Sigma \beta_{t}=l-m}\left(\frac{\partial^{l} x}{\partial t_{1}^{m_{1}+\beta_{1}} \ldots \partial t_{n}^{m_{n}+\beta_{n}}}\right)^{2}\right]^{p / 2} d t+ \\
& +\sum_{k=0}^{l-m-1}\left[\sum_{\Sigma \beta_{i}=k}\left(L_{\beta_{1} \ldots \beta_{n} ; \nu}(x)\right)^{2}\right]^{p / 2}
\end{aligned}
$$

where we have set

$$
L_{\beta_{1} \ldots \beta_{n}}(x)=M a_{\beta_{1} \ldots \beta_{n ; v}}^{(m)}
$$

and where the $a_{\beta_{1} \ldots \beta_{n ; \nu}}^{(m)}$ are obtained as follows: if $P \in S^{l}$ is the polynomial occurring in the representation (A.2) of $x$, then the $a_{\beta_{1} \ldots \beta_{n} ; \nu}^{(m)}$ are the coefficients of the polynomial

$$
P_{l-m ; v}=\frac{\partial^{m} P}{\partial t_{1}^{m_{1}} \ldots \partial t_{n}^{m_{n}}} \in S^{l-m} .
$$

We claim that the $L_{\beta_{1} \ldots \beta_{n ; v}}$ defined by (A.10) are linear bounded functionals on $W_{p}^{l}(I)$. Indeed the linearity is obvious. As to the boundedness, we know already that the $a_{\beta_{1} \ldots \beta_{n ; v}}^{(m)}$ are linear and bounded on $W_{p}^{l-m}(I)$, i.e. that there exists a $C>0$ such that

$$
\left|a_{\beta_{1} \ldots \beta_{n}}\left(x_{v}^{(m)}\right)\right| \leqq C\left\|x_{v}^{(m)}\right\|_{W_{p}^{t-m}}=C\left\{\left\|x_{v}^{(m)}\right\|_{p}+\left\|x_{v}^{(m)}\right\|_{l-m, p}\right\} .
$$

Now the $p^{\text {th }}$ power of $\left\|x_{v}^{(m)}\right\|_{t-m, p}$ is by definition precisely the integral at the right member of (A.9), and this integral clearly $\leqq\|x\|_{l, p} \leqq\|x\|_{W_{p}^{l}(I)}$. On the other hand $\left\|x_{v}^{(m)}\right\|_{p} \leqq$ Const. $\|x\|_{w_{p}^{l}(I)}$ which inequality follows from [13; theorem, p. 69] 
in the same way as the inequality (4.3). These inequalities together with (A.12) and (A.10) prove our assertion.

We now consider the cube $I_{\delta} \subset E^{n}$ of side length $\delta<1$ with center $\vartheta^{0}=\left(\vartheta_{1}^{0} \ldots \vartheta_{n}^{0}\right)$, and parallel to the cube $I$. Setting

$$
\vartheta_{i}=\vartheta_{i}^{0}+\delta t, \quad \xi(\vartheta)=x(t) \text { where } \vartheta=\left(\vartheta_{1} \ldots \vartheta_{n}\right),
$$

and taking into account that the Jacobian

$$
\frac{\partial\left(t_{1}, \ldots, t_{n}\right)}{\partial\left(\vartheta_{1}, \ldots, \vartheta_{n}\right)}=\delta^{-n}
$$

and that

$$
\frac{\partial^{m} x}{\partial t_{1}^{m_{1}} \ldots \partial t_{n}^{m_{n}}}=\delta^{m} \frac{\partial^{m} \xi}{\partial \vartheta_{1}^{m_{1}} \ldots \partial \vartheta_{n}^{m_{n}}},
$$

we see that (A.9) written in the new variables becomes

$$
\begin{aligned}
& \int_{I_{\delta}}\left|\frac{\partial^{m} \xi}{\partial \vartheta_{1}^{m_{l}} \ldots \partial \vartheta_{n}^{m_{n}}}\right|^{p} d \vartheta \\
& \quad \leqq M^{p} \delta^{(l-m) p} \int_{I_{\delta}}\left[\sum_{\Sigma \beta_{i}=l-m}\left(\frac{\partial^{l} \xi}{\partial \vartheta_{1}^{m_{1}+\beta_{1}} \ldots \partial \vartheta_{n}^{m_{n}+\beta_{n}}}\right)^{2}\right]^{p / 2} d \vartheta+ \\
& \quad+\delta^{-m p} \sum_{k=0}^{l-m-1}\left[\sum_{\Sigma \beta_{i}=k}\left(\lambda_{\beta_{1} \ldots \beta_{n}}(\xi)\right)^{2}\right]^{p / 2}
\end{aligned}
$$

where

$$
\lambda_{\beta_{1} \ldots \beta_{n}}(\xi)=\delta^{n / p} L_{\beta_{1} \ldots \beta_{n, v}}(x) .
$$

We claim the $\lambda_{\beta} \ldots \beta_{n}$ are bounded linear functionals on $W_{p}^{l}\left(I_{\delta}\right)$ with bounds independent of $\delta$. Since we know that the $L_{\beta_{1} \ldots \beta_{n}}$ are bounded linear functionals on $W_{p}^{l}(I)$, we have to prove: if $L(x)$ is a bounded linear functional on $W_{p}^{l}(I)$, then

$$
\lambda(\xi)=\delta^{n / p} L(x), \quad 0<\delta<1
$$

is a bounded linear functional on $W_{p}^{l}\left(I_{\delta}\right)$ with a bound independent of $\delta$.

We omit the obvious proof of the linearity. Let $\gamma$ be a bound for $L$. Then

$$
\begin{aligned}
|\lambda(\xi)|^{p} & =\delta^{n}|L(x)|^{p} \leqq \delta^{n} \gamma^{p}\|x\|_{W_{p}^{l}(I)}^{p} \\
& =\delta^{n} \gamma^{p}\left\{\int_{I}|x|^{p} d t+\int_{I}\left[\sum_{\Sigma \alpha_{l}=l}\left(\frac{\partial^{l}}{\partial t_{1}^{\alpha_{1}} \ldots \partial t_{n}^{\alpha_{n}}}\right)^{2}\right]^{p / 2} d t\right\} \\
& =\delta^{n} \gamma^{p}\left\{\delta^{-n} \int_{I_{\delta}}|\xi|^{p} d \vartheta+\delta^{-n} \delta^{l p} \int_{I_{\delta}}\left[\sum_{\Sigma \alpha_{l}=l}\left(\frac{\partial^{l} \xi}{\partial \vartheta_{1}^{\alpha_{1}} \ldots \partial \vartheta_{n}^{\alpha_{n}}}\right)^{2}\right]^{p / 2} d \vartheta\right\},
\end{aligned}
$$

where we again used (A.14) and (A.15). This inequality remains valid if in its right hand member we replace the factor $\delta^{l p}$ of the second integral by 1 . But then the right hand member becomes $\gamma^{p}\|\xi\|_{W_{p}^{t}\left(I_{\delta}\right)}$ which proves that $\gamma$ is a bound for $\lambda$.

We now return to the Sobolev domain $\Omega$ considered in theorem 4.2 and suppose that $I_{\delta} \subset \Omega$. Then the following facts are easily verfied: if we map the 
element $x \in W_{p}^{l}\left(I_{\delta}\right)$ on $\tilde{x}=\tilde{x}(t)$ defined by

$$
\begin{aligned}
\tilde{x}(t) & =x(t) & \text { for } & t \in I_{\delta} \\
& =0 & \text { for } & t \in \Omega-I_{\delta},
\end{aligned}
$$

we obtain an isometric imbedding of $W_{p}^{l}\left(I_{\delta}\right)$ in $W_{p}^{l}(\Omega)$, and $W_{p}^{l}\left(I_{\delta}\right)$ thus considered as subset of $W_{p}^{l}(\Omega)$ is a closed linear subspace of $W_{p}^{l}(\Omega)$. Consequently a linear bounded functional $\lambda$ on $W_{p}^{l}\left(I_{\delta}\right)$ can be extended to a bounded linear functional on $W_{p}^{l}(\Omega)$. Therefore we may and will consider the $\lambda_{\beta_{1} \ldots \beta_{n}}$ appearing in (A.16) as bounded linear functionals on $W_{p}^{l}(\Omega)$.

Now let $I_{\delta}^{j}(j=1, \ldots, N)$ and $\Omega_{N}$ be as described in the statement of theorem 4.2. If then $\xi \in W_{p}(\Omega)$, then the restriction of $\xi$ to $I_{\delta}^{j}$ belongs to $W_{p}^{l}\left(I_{\delta}^{j}\right)$. Therefore (A.16) holds for each $I_{\delta}^{j}$. Since the $\lambda_{\beta_{1} \ldots \beta_{n}}$ now depend on $j$ we use the notation $\lambda_{\beta_{1} \ldots \beta_{n}}^{j}$ for them. If we add the inequalities thus obtained over $j$ from 1 to $N$, we obtain the asserted inequality (4.1).

\section{Appendix B}

Another application of theorem 4.3.

Theorem. Let $B$ be a bounded set in $W_{p}^{l}(\Omega)$, and let ${ }_{\rho} x(\rho=1,2, \ldots)$ be a sequence of elements of $B$. Then there exists a subsequence $\rho_{i}$, such for $m=0,1, \ldots, l-1$, $v=1,2, \ldots, \sigma_{m}$ the sequence of derivatives ${ }_{p_{1}} x_{v}^{(m)},{ }_{p_{2}} x_{v}^{(m)}, \ldots$ converges strongly in $L_{p}$.

Proof. Since $B$ is bounded, there exists an $R$ such that $B \subset B_{R}$. Since $B_{R}$ is weakly compact and therefore closed in the weak topology of $W_{p}^{l}$, we have $\bar{B} \subset \bar{B}_{R}=B_{R}$ where the bar denotes closure in the weak topology. As a closed subset of the compact set $B_{R}, \bar{B}$ is also compact in the weak topology. It follows by a well known theorem (see $e . g .[6 ;$ p.430]) that $B$ is weakly sequentially compact, which means by definition: every sequence ${ }_{\rho} x$ in $B$ contains a subsequence $\rho_{t} x$ to which there exists an ${ }_{0} x \in W_{p}^{l}$ such that

$$
\lim _{i \rightarrow \infty} \lambda\left(\rho_{i} x\right)=\lambda\left({ }_{0} x\right)
$$

for every bounded linear functional $\lambda$ on $W_{p}^{l}$. Now ${ }_{\rho_{i}} x-{ }_{\rho_{j}} x \in B_{2 R}$ since ${ }_{\rho} x \in B \in B_{R}$ for all $\rho$. We see therefore from theorem 4.3 that $\left\|_{\rho_{t}} x-{ }_{\rho_{j}} x\right\|_{p}^{p}$ is majorized by the right member of (4.11) with $\sigma L_{1}$ replaced by 1 , and $x-a$ by $\rho_{i} x-{ }_{\rho_{j}} x$. Arguing as in the lines following (4.11), we see that to given positive $\varepsilon$ we can choose first $\eta$ and then $\delta$ in such a way that the first two terms of the modified right member of (4.11) together are not greater than $2 \varepsilon / 3$. It now follows from (B.1) that there exists an $i_{0}$ such that the third term becomes less than $\varepsilon / 3$ for $i$ and $j$ greater than $i_{0}$.

Remark. If $B$ is closed in the weak topology, then ${ }_{0} x \in B$. Sufficient for the weak closure of $B$ is that $B$ is convex and strongly closed (see e.g. $[6 ;$ p.422]).

\section{Bibliography}

[1] BourbaKi, N., Éléments de mathématique, livre V, Espaces vectoriels topologiques. Paris: Hermann \& Co. 1953.

[2] BRowDER, F.E., Non linear elliptic boundary value problems. Bull. A.M.S. 69, 862-874 (1963). 
[3] BRowDER, F.E., Variational methods for nonlinear elliptic eigenvalue problems. Bull. A.M.S. 71, 176-183 (1965).

[4] Courant, R., \& D. Hilbert, Methoden der mathematischen Physik II. Berlin: J. Springer 1937.

[5] DAY, M.M., Uniform convexity III. Bull. A. M.S. 49, 744-750 (1943).

[6] Dunford, N., \& J. T. SchwarTz, Linear Operators, part I, General theory 1958. New York: Interscience Publishers Inc.

[7] FrIedrichs, K.O., On the differentiability of solutions of linear elliptic differential equations. Communications on Pure and Applied Mathematics 6, 299-326 (1953).

[8] Graves, L.M., On the existence of absolute minima in space problems of the calculus of variations. Annals of Mathematics 28, 153-170 (1926/27).

[9] Minty, G.J., On the monotonicity of the gradient of a convex function. Pacific Journal of Mathematics 14, 243-247 (1964).

[10] Rothe, E.H., Gradient mappings and extrema in Banach spaces. Duke Math. Journal 15, $421-431$ (1948).

[1I] Rothe, E.H., A note on the Banach spaces of Calkin and Morrey. Pacific Journal of Mathematics 3, 493-499 (1953).

[12] Smirnov, W.I., Lehrgang der Höheren Mathematik, part V. Berlin: Deutscher Verlag der Wissenschaften 1962 (translated from the Russian edition of 1959).

[13] Sobolev, S.L., Applications of functional analysis in mathematical physics. A.M.S. 1963 (translated from the Russian edition of 1950).

The University of Michigan

Ann Arbor, Michigan

(Received August 30, 1965) 